6

\title{
Biotin-Ligand Complexes With Streptavidin Quantum Dots for In Vivo Cell Labeling of Membrane Receptors
}

\author{
Diane S. Lidke, Peter Nagy, Thomas M. Jovin, and Donna J. Arndt-Jovin
}

\begin{abstract}
Summary
The unique fluorescence properties of quantum dots (QDs), particulary their large extinction coefficients and photostability, make them ideal probes for tracking proteins in live cells using real-time visualization. We have shown that QDs conjugated to epidermal growth factor act as functional ligands for their receptor, erbB1. Here, we describe protocols for (1) conjugation of streptavidin-QDs to biotinylated ligand, (2) formation of ligand-QD-receptor complexes, and (3) quantification of binding and internalization of receptor complex using both high-resolution fluorescence microscopy and flow cytometry.
\end{abstract}

Key Words: Epidermal growth factor; EGF; epidermal growth factor receptor; EGFR; erbB1; endocytosis; biotin; streptavidin.

\section{Introduction}

The erbB family of receptor tyrosine kinases includes erbB1 (the classical epidermal growth factor receptor, EGFR), erbB2, erbB3 and erbB4 (1). Binding of epidermal growth factor (EGF) to the extracellular domain of erbB1 leads to auto- and transactivation of cytoplasmic protein kinase domains in response to the formation (2) or reorientation (3-5) of homo- and hetero-associated receptor tyrosine kinase monomers. This activation of the receptor initiates signaling cascades, which control a variety of cellular processes such as DNA replication and division (1,6). ErbB1 activation results in clathrin-mediated endocytosis via coated pits, covalent modification of the receptor, and endosomal trafficking resulting in both downregulation through proteosomal degradation and recycling to the cell membrane. The proper function of erbB1 in the cell is critical, given that overexpression or mutations of the erbB1 are implicated in many types of cancer $(1,7)$. 
We have shown that quantum dots (QDs) conjugated to EGF act as functional ligands for erbB1, and that their unique fluorescence properties provide the means for prolonged real-time visualization of the multiple steps in signaling mechanisms in living cells $(\mathbf{8 , 9 )}$. In this chapter we outline procedures for binding biotinylated ligands to commercial streptavidin-conjugated QDs for specific labeling of growth factor receptors with these ligands and for quantitative measurements of the receptor complexes by both fluorescence microscopy and flow cytometry.

\section{Materials}

1. Phosphate-buffered saline (PBS): $137 \mathrm{~m} M \mathrm{NaCl}, 2.7 \mathrm{~m} M \mathrm{KCl}, 7.9 \mathrm{~m} M \mathrm{Na}_{2} \mathrm{HPO}_{4}$, and $1.5 \mathrm{n} M \mathrm{KH}_{2} \mathrm{PO}_{4}, \mathrm{pH} 7.3$. Autoclave before use and store at room temperature. PBS/bovine serum albumin (BSA): add $0.1 \%$ BSA just before use.

2. Tyrode's buffer without glucose: $135 \mathrm{~m} M \mathrm{NaCl}, 10 \mathrm{~m} M \mathrm{KCl}, 0.4 \mathrm{~m} M \mathrm{MgCl}_{2}$, $1 \mathrm{~m} M \mathrm{CaCl}_{2}$, and $10 \mathrm{~m} M$ HEPES, $\mathrm{pH}$ 7.2. Autoclave before use and store at room temperature. Tyrode's plus: add $20 \mathrm{~m} M$ glucose and $0.1 \%$ BSA just before use.

3. Streptavidin-conjugated QDs (SAvQD) from Invitrogen (Eugene, OR, www. probes.com).

4. LabTek eight- or two-well cover slip chambers from Nunc (Rochester, NY).

5. Cover slips, acid washed and sterilized.

6. Cell culture medium appropriate for the particular cell line. Various commercial vendors.

7. Emission filters for specific QD emission wavelengths, $20 \mathrm{~nm}$ FWHM Chroma (Brattleboro, VT).

8. Biotinylated EGF and EGF from Molecular Probes (Invitrogen, www.probes.com).

9. Paraformaldehyde (PFA) fixative: PFA, analytical grade. Freshly prepared $4 \%$ solution in PBS. A 40\% solution can be prepared and kept in small aliquots at $-20^{\circ} \mathrm{C}$. Thaw at $50^{\circ} \mathrm{C}$ and immediately dilute in PBS before use.

10. Tris-saline buffer: $125 \mathrm{mM} \mathrm{NaCl}, 25 \mathrm{mM}$ Tris-HCl, $\mathrm{pH}$ 7.3.

11. Trypsin solution for releasing adherent cells from their substrates: sterile solution of $0.5 \mathrm{mg} / \mathrm{mL}$ trypsin in PBS containing $1 \mathrm{~m} M$ EDTA.

12. Acid wash buffer: $0.5 \mathrm{M} \mathrm{NaCl}$ and $0.1 \mathrm{M}$ glycine- $\mathrm{HCl}, \mathrm{pH} 2.5$.

\section{Methods}

\subsection{Making Biotin-Ligand Linked SAvQD}

\subsubsection{Ratio of Biotinylated Ligand to QDs}

The present formulation of the QDs from Quantum Dot Corp. includes a polyethylene glycol 2000 outer layer to which 5-10 streptavidin molecules per QD are covalently linked. This allows the stochiometry of the ligand-QD complex to be varied. It is preferable to use monovalent biotinylated ligands where the biotin is conjugated to a single known residue or position. In this way the properties of the ligand-QD complex can be better controlled and standardized, 
and potential cross-linking of the QDs via the ligand is avoided. By mixing SAvQDs, biotinylated ligand, and free biotin at different molar ratios, one can create QDs with different numbers of attached ligands. We assume a Poisson distribution. To create primarily monovalent biotin conjugates, mix a 10-fold molar excess of SAvQDs with the biotinylated ligand.

\subsubsection{Preformed Complexes}

1. Dilute SAvQD to $20 \mathrm{n} M$ in PBS/BSA.

2. Dilute biotinylated ligand to the appropriate desired molarity in PBS/BSA.

3. Add SAvQDs to ligand and mix with a micropipet.

4. Incubate at $4^{\circ} \mathrm{C}$ for at least $30 \mathrm{~min}$ with gentle agitation or rotation (see Note $\mathbf{1}$ ).

\subsubsection{Purification of Ligand-QD Conjugates}

After the ligand-QD complex is formed, unbound ligands that are smaller than the QDs can be removed from the conjugate by passing the QDs through a size exclusion column. For SAvQDs described, Sephadex G-25 medium grade gel filtration is recommended; the SAvQDs will elute in the void volume. Use a 1:20 ratio of sample to column volume for good separation. Spin columns can be used for sample volumes of $50 \mu \mathrm{L}$ or less.

1. Swell and pour the Sephadex according to the manufacturer's instructions.

2. Equilibrate the column in PBS by washing with at least three column volumes.

3. Allow the buffer to just enter the top of the gel bed before adding the sample. Avoid air bubbles.

4. Add the sample to the top of the gel bed, allow the sample to enter the gel, and add PBS to elute the sample.

5. Collect the fractions containing the QDs.

\subsection{In Vivo, In Situ Labeling of Cells With Ligand-QD Conjugates for Fluorescence Microscopic Imaging}

\subsubsection{Plating cells-Cover Slips and Cover Slip Chambers}

Logarithmically growing cells are replated in complete culture medium at the appropriate densities 1 or $2 \mathrm{~d}$ prior to the experiment. In the case of experiments with growth factor receptors, the cells are serum-starved for 16-24 h prior to the experiment to reduce signaling induced by the serum in the medium.

\subsubsection{Labeling Cell Surface Receptors With Preformed Ligand-QD Complex}

1. Wash cells once with Tyrode's plus and maintain in this buffer for the experiment.

2. Place the cover slip chamber on the microscope stage and equilibrate to the desired temperature.

3. Dilute the ligand-SAvQD complex (in this case EGF-QD) to twice the final concentration in Tyrode's plus (see Note 2). 
4. Add an equal volume of EGF-QD in Tyrode's plus to the cells in the well under observation to a final concentration of $5-200 \mathrm{pM}$.

5. Start imaging.

\subsubsection{Labeling Cell Surface Receptors in a Two-Step Procedure}

In some situations the preformed complex may not be functional or the system may not respond properly with the preformed complex because of (1) steric hindrance by the SAvQD during ligand binding or (2) SAvQD inhibition of a conformational change of the ligand required for binding. In addition, ligands with multiple biotins can cause aggregation of SAvQDs. Thus, if one does not see binding with the preformed complex or observes aggregation of SAvQDs in the onestep protocol, the following two-step protocol may be useful. We have successfully used this procedure with both the EGF and the transferrin receptors $(8,10)$.

1. Wash cells once with ice cold Tyrode's plus and keep in this buffer for the experiment. Put cells on ice.

2. Incubate cells with biotinylated-ligand (in this case $1 \mathrm{n} M$ biotin-EGF, see Note 3) for 5-10 min.

3. Wash with ice cold Tyrode's plus several times.

4. Add cold $200 \mathrm{p} M$ SAvQD for 5-15 min.

5. Wash the cells several times with cold Tyrode's plus.

6. Place cells on the microscope stage with appropriate temperature control and image.

7. For snapshots of the behavior of the ligand-QD complex over time, incubate cover slips at the appropriate temperatures and times and fix in 4\% PFA for 15 min on ice to prevent redistribution of the QDs. After fixation, wash the cover slips with Tris-saline buffer for 10 min several times and mount in PBS for microscopy.

\subsubsection{In Vivo Visualization and Quantitation of Ligand-QD Binding by Fluorescence Confocal Microscopy}

Quantitative binding and/or internalization kinetic plots can be generated from the QD signals if cells express a membrane protein fused to a visible fluorescent protein (GFP or any VFP) or are stained with a membrane specific probe.

1. Grow cells in an eight-well LabTek chambers as previously described.

2. Wash cells once with Tyrode's plus and keep in 200-250 $\mu \mathrm{L}$ of Tyrode's plus for imaging.

3. Begin acquisition of a time series with single or multiple focal planes.

4. Typically, a $63 \times$ or $40 \times 1.2 \mathrm{NA}$ water immersion objective is recommended. Simultaneous excitation of GFP and QD605 can be achieved at $488 \mathrm{~nm}$. An imaging system with two detectors, e.g., Zeiss LSM510-META, allows the simultaneous collection of GFP and QD signals with appropriate filters (in this case, 520/20 bandpass and 585 longpass, respectively).

5. After several time-points are acquired, add the preformed EGF-QD complexes to the buffer (1:1 dilution) without disrupting the time series. 


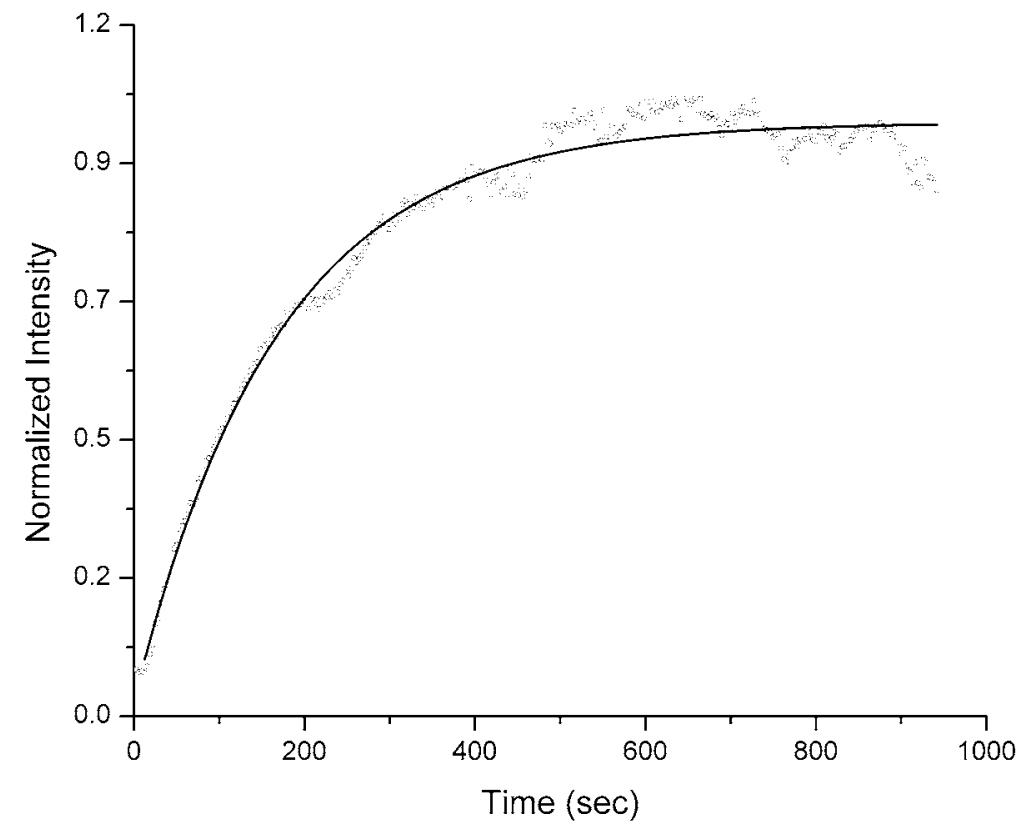

Fig. 1. Quantitation of epidermal growth factor-quantum dots (EGF-QDs) binding to Chinese hamster ovary cells expressing erbB1-eGFP. Plot shows the change in QD intensity inside the membrane mask over time. The binding of the EGF-QDs to the cell surface follows an exponential course.

6. Analysis (8).

7. Background subtraction from both channels is performed.

8. The membrane region of the cell is segmented by a global threshold in the green channel and the mask is dilated according to a pixel width corresponding to the membrane circumference.

9. The average QD intensity (from the red channel) in the membrane mask is plotted over time (see Fig. 1).

\subsubsection{Two-Color QD "Chase" Experiment}

Multicolor EGF-QDs may be used to track endocytosis of the receptor-ligand complex and to follow subsequent steps in time. Two different colors of ligandQDs are delivered sequentially (see Fig. 2).

1. The day before the experiment, plate cells on cover slips if taking fixed cell timepoints or in Lab-Tek cover slip chambers for live cell imaging.

2. Prepare two batches of QD-ligand, with separated emission spectra, such as SAvQD525 and SAvQD605.

3. Wash the cells in Tyrode's plus buffer and keep in this buffer. 

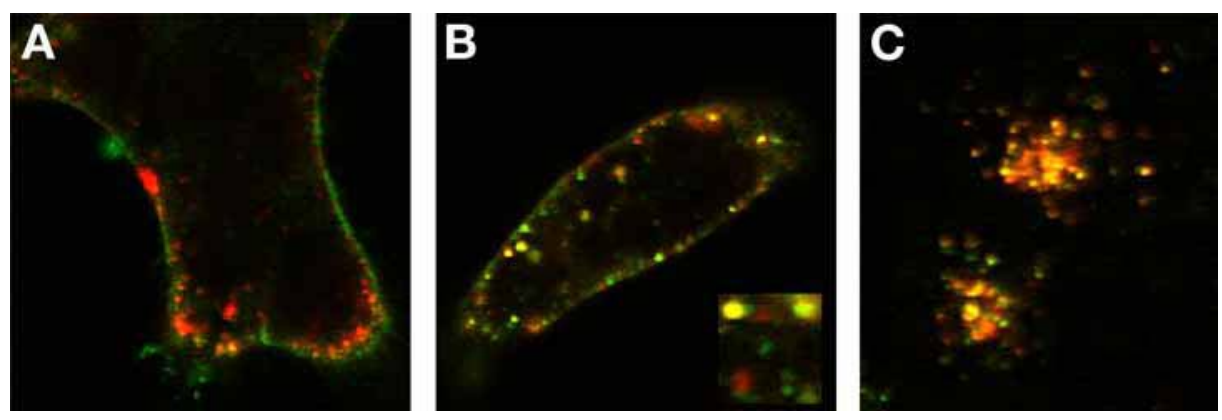

Fig. 2. Two-color quantum dot (QD) "chase" experiment of epidermal growth factor (EGF)-QDs binding to A431 cells expressing erbB1. (A) EGF-QD605 (red) was bound to ErbB1 on A431 cells for 3 min at room temperature. Cells were washed and incubated for $5 \min 37^{\circ} \mathrm{C}$ to induce internalization. EGF-QD525 (green) was then added and incubated for $8 \mathrm{~min}$ at $37^{\circ} \mathrm{C}$. Early endosomes are red only, indicating that the EGFQD605 has internalized, whereas the EGF-QD525 remains predominantly external. (B) Twenty minutes later. Several different classes of endosomes are observed. The endosomes contain red only, green only or both QD colors (yellow, see inset), indicating that early, sorting and late endosomes are loaded. (C) Two hours later. Although all vesicles show red and green signals, clear differences in QD populations are seen in the content of late endosomes and lysosomes.

4. Incubate the cells in a non-saturating concentration $(<200 \mathrm{p} M$ in the case of EGF-QD) of the first QD-ligand (QD605) for $5-10$ min at $37^{\circ} \mathrm{C}$ while taking time-lapse images on a confocal microscope or incubate cover slips in a moist chamber for samples to subsequently fix.

5. Wash away excess QD-ligand by two changes of Tyrode's plus.

6. Incubate at $37^{\circ} \mathrm{C}$ for at least 5 min to clear the membrane of ligand-QD (image in vivo).

7. Add $1 \mathrm{n} M$ of ligand of another color, EGF-QD (QD525), and incubate at $37^{\circ} \mathrm{C}$ for desired time in vivo with imaging or for desired fixed time-points.

8. For fixed samples, wash cover slips in Tyrode's buffer and fix at $4{ }^{\circ} \mathrm{C}$ for $15 \mathrm{~min}$ in 4\% PFA (see Subheading 3.2.3.).

9. Wash with cold Tris-saline several times.

10. Antibody staining for activated erbB1, endocytic markers, or other cell components can be carried out at this point.

11. Mount cover slips onto a drop of PBS on a microscope slide and seal with nail polish. Image in fluorescence microscope using appropriate filters.

\subsection{Quantitative Measurement of EGF-QD Binding and Internalization Using Flow Cytometry}

Many properties of QDs, including their brightness and extreme photostability, make them very valuable for time-lapse fluorescence microscopy. However, 
a QD-labeled ligand or antibody is increased in size by 10-20 $\mathrm{nm}$ per bound QD. Therefore, carefully planned control experiments are necessary to establish that the QD does not interfere with the normal biological function of the ligand or antibody. Flow cytometry is an indispensable method for quantitation of binding and internalization data with high statistical precision. The following protocol can be used to show specific and saturable binding of EGF-QD to erbB1 on the cell surface, and demonstrate its uncompromised biological activity by monitoring internalization of the acceptor-ligand complex.

\subsubsection{EGF-QD Specifically Binds to a Saturable Binding Site in the Membrane of erbB1-Expressing Cells}

1. Prepare preformed EGF-QD at a ratio of $1: 1$ as described in Subheadings 3.1.2.-3.1.3.

2. Trypsinize A431 cells and resuspended in PBS/BSA at a density of $5 \times 10^{6} / \mathrm{mL}$, and store on ice (see Note 4).

3. Prepare a twofold dilution series of EGF-QDs: make $1000 \mu \mathrm{L}$ of the highest concentration of EGF-QD $(100 \mathrm{n} M)$ in PBS/BSA in a flow cytometry tube (see Note 5). Thorough mixing is very important. The tubes are kept on ice during the entire labeling procedure. Five hundred microliter is pipetted from this solution to another tube to which $500 \mu \mathrm{L}$ of PBS/BSA is added yielding the second highest concentration $(50 \mathrm{nM})$ of EGF-QD. This procedure is repeated until the lowest concentration is reached. The result is a dilution series of tubes filled with $500 \mu \mathrm{L}$ of different concentrations of the ligand on ice (see Note 6).

4. Add $20 \mu \mathrm{L}$ of the cell suspension to each ligand solution and mix thoroughly (see Note 7). Incubate the cells on ice for $60 \mathrm{~min}$ with regular mixing.

5. Measure the samples on a flow cytometer without washing (see Note 8). An unlabeled cell sample is also included in the measurement, the fluorescence intensity of which is subtracted from the mean fluorescence intensities of each sample. The corrected fluorescence intensities are plotted as a function of ligand concentration, and the characteristics of ligand binding are analyzed.

\subsubsection{Binding of Streptavidin-Coated QDs to Cells Labeled With Different Molar Ratios of EGF and Biotin-EGF}

Results of the previous experiments showed that EGF-QD binds to a saturable binding site on the surface of A431 cells. Strong evidence for specific binding is provided by competitive inhibition by another ligand. Activation of erbB1 requires the binding of two EGF molecules to a dimer of the erbB1 receptor $(2,11)$. The relatively large size of QDs requires one to show unambiguously that the ligand-QD is functional. Furthermore, if erbB1 molecules are at high density on the cell surface of A431 cells, a bound QD may sterically hinder binding to a neighboring receptor. To rule out size or steric hindrance by the QDs, we labeled cells with different molar ratios of EGF and biotinylated-EGF, followed by SAvQD, and 


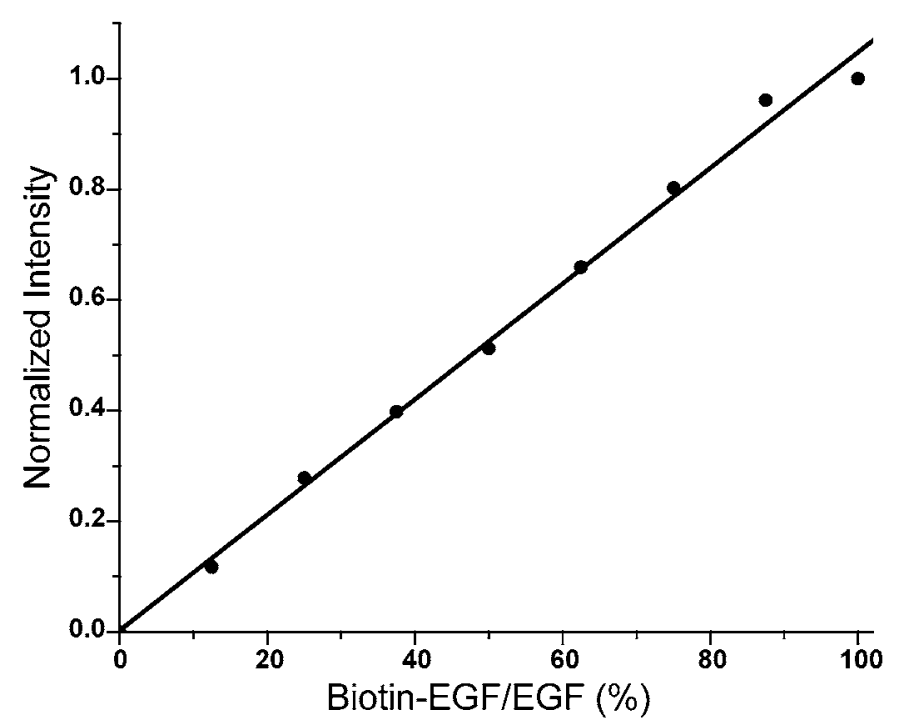

Fig. 3. Binding of streptavidin-cojugated quantum dots (SAvQDs) to cells labeled with different molar ratios of biotinylated- and non-biotinylated-EGF. A431 cells were incubated in the presence of different molar ratios of biotin-EGF and EGF for $60 \mathrm{~min}$ followed by labeling with SAvQDs. The fluorescence intensity of the samples (empty circles) was measured using flow cytometry. The correlation coefficient of a linear fit to the data (straight line) was $\mathrm{R}=0.99$. (Reproduced with permission from ref. 8 .)

determined whether the fluorescence intensities of the samples were linearly proportional to the molar fraction of biotin-EGF. The higher the molar fraction of biotinylated-EGF, the higher the probability of finding two biotinylated-EGF molecules close to each other, e.g., binding to two erbB1 proteins in a dimer. This would result in leveling off of the fluorescence intensity curve at high biotin-EGF concentrations. The fact that we found a strictly linear relationship implies that binding of streptavidin-coated QDs is not sterically limited (see Fig. 3).

1. Trypsinize A431 cells and resuspend in PBS/BSA at a density of $2 \times 10^{6} / \mathrm{mL}$. Store on ice (see Note 4).

2. Prepare $100 \mathrm{n} M$ stock solutions of biotin-EGF and EGF in PBS/BSA. Keep the solutions on ice.

3. Prepare a $20 \mathrm{n} M$ stock solution of SAvQD in PBS/BSA and store on ice.

4. Prepare a series of samples (total volume of each is $50 \mu \mathrm{L}$ ) in which the molar ratio of biotin-EGF changes from 0 to $100 \%$, e.g. $0,12.5,25,37.5,50,62.5,75$, $87.5,100 \%)$. The total concentration of EGF (biotinylated + nonbiotinylated) is $100 \mathrm{n} M$ in each sample.

5. Add $50 \mu \mathrm{L}$ of cell suspension to each tube and mix thoroughly. Incubate the samples on ice for 30 min with regular shaking. 
6. Layer $1 \mathrm{~mL}$ of fetal calf serum under the cell suspension and centrifuge the cells at $4^{\circ} \mathrm{C}$. Discard the supernatant.

7. Add $300 \mu \mathrm{L}$ of the $20 \mathrm{n} M$ QD solution to each pellet and incubate the samples on ice for 30 min with regular shaking (see Note 9).

8. Without washing, measure the sample intensities using a flow cytometer.

\subsubsection{Quantitative Measurement of EGF-QD Internalization}

Although microscopy experiments can measure the internalization of ligandQD in a qualitative or semi-quantitative manner with subcellular resolution, flow cytometry is indispensable for obtaining a quantitative description of the process.

1. Couple biotin-EGF to SAvQDs at a molar ratio of 6:1 as described in Subheading 3.1.2.

2. Trypsinize A431 cells and resuspended at a density of $10^{6} / \mathrm{mL}$ in Tyrode's buffer plus.

3. Add $1 \mathrm{n} M$ EGF-QD to the sample and incubate at $37^{\circ} \mathrm{C}$ with regular shaking.

4. Take $600-\mu \mathrm{L}$ samples at $0,5,10,20$, and $30 \mathrm{~min}$.

5. Add $5 \mathrm{~mL}$ of acid wash buffer to half of the sample, and incubate on ice for $5 \mathrm{~min}$.

6. Wash with a large excess of PBS and resuspend cells in $300 \mu \mathrm{L}$ Tyrode's plus.

7. Measure the fluorescence intensities of the acid washed sample and the non-acidtreated sample by flow cytometry to obtain the fraction of internalized ligand (see Note 10).

\subsection{Other Types of In Vivo Labeling of Cells With Quantum Dots}

QDs with many different types of conjugation are commercially available. Additionally, manufacturers have developed kits for alternative methods of biocoupling QDs. We recommend the following recent papers and reviews for additional cell labeling techniques with quantum dots (12-15).

\section{Notes}

1. Thoroughly mix the SAvQDs and ligands by pipetting up and down several times. Mix in equal volumes so that the stoichiometry does not vary throughout the solution at the time of mixing.

2. Dilute the ligand-SAvQD complex from PBS into Tyrode's plus just before use because the SAvQDs aggregate with time in buffers containing divalent cations.

3. In performing similar experiments on another system than the EGFR, the researcher should adjust the concentration of ligand and incubation times according to the binding constant of the receptor or protein-ligand interaction.

4. The cell membrane is in a constant state of recycling. Antibodies and ligands typically induce the internalization of their receptor, and newly synthesized receptors continuously reach the plasma membrane by exocytosis. If cells are labeled at a temperature permissive to endo- and exocytosis, cells engulf EGF-QD 
receptors, and the freshly exocytosed receptors will also bind EGF-QD from the continued presence of the label in the extracellular space. This results in a higher level of EGF-QD binding than would be expected based on the original number of receptors on the cell surface, giving rise to binding that is difficult to saturate. Therefore, both the cell suspension and the ligand dilution series are kept on ice during the whole duration of the experiment, and all solutions are prepared in flow cytometry tubes to minimize the number of times a solution has to be transferred from one tube to another. Although flow cytometers are usually not thermostated, if the tubes are kept on ice until measurement, ligand internalization is minimized.

5. The amount of receptor-bound ligand is usually negligible compared with the total amount. However, if a high-affinity ligand is investigated and the labeling volume is minimized, the decreased concentration of free ligand in the extracellular space (called ligand depletion) cannot be overlooked. For example, if $10^{5}$ cells expressing $10^{6}$ receptors/cell are labeled in the presence of $10 \mathrm{n} M$ ligand in a volume of $500 \mu \mathrm{L}$, the number of bound ligands is $10^{5} \times 10^{6}=10^{11}$ assuming saturation. The total number of added ligands is $10 \times 10^{-9} \mathrm{M} \cdot 500 \times 10^{-6} \mathrm{~L} \times 6 \times 10^{23} \mathrm{1} / \mathrm{mol}=3 \times 10^{12}$. In this case $33 \%$ of the total ligand amount is bound, severely distorting the calculations. As a rule of thumb, 5\% ligand depletion is usually considered negligible. Beyond this limit either the real free-ligand concentration has to be determined, or corrections have to be introduced in the calculation, the details of which are beyond the scope of this chapter.

6. The ligand can also be depleted by binding to the wall of the labeling tube which can be minimized by carrying out the experiment in the presence of $0.1-0.5 \%$ $(w / v)$ BSA. In addition, the same number of cells are labeled in identical volumes to ensure similar conditions (e.g., a constant number of total binding sites).

7. Dilution of the ligand after adding $20 \mu \mathrm{L}$ cell suspension to $500 \mu \mathrm{L}$ ligand solution is either neglected or can be corrected for in the following way: $c_{\text {corr }}=c_{\text {orig }} \cdot 500 / 520$, where $c_{\text {corr }}$ and $c_{\text {orig }}$ are the concentrations of the ligand in the diluted and undiluted solutions, respectively.

8. Ligand dissociation starts immediately when the concentration of ligand drops in the extracellular solution. Therefore, we suggest that samples not be washed before labeling. In the case of high-affinity ligands saturating their binding site at a low concentration, fluorescence of the extracellular ligands is negligible. In addition, flow cytometers are equipped with a constant background subtraction algorithm, reliably accounting for the contribution of extracellular fluorescence even in the case of low-affinity ligands.

9. The number of erbB1 proteins in $10^{5}$ labeled A431 cells is $10^{5} \times 2 \times 10^{6}=2 \times 10^{11}$ corresponding to $2 \times 10^{11} / 6 \times 10^{23} / 300 \mu \mathrm{L}=1.1 \mathrm{n} M$ mean receptor concentration. The concentration of QD was chosen to be $20 \mathrm{n} M$ to ensure an approx 20-fold excess of unbound QDs.

10. Because acid washing removes non-covalently-bound molecules from the cell surface, the fluorescence intensity of acid-washed samples will represent the internalized pool of QD-labeled ligands. 


\section{Acknowledgments}

This work was funded by EU FP5 Grants QLRT-2000-02278 and QLG1-CT2000-01260 awarded to T.M.J and the Max Planck Gesellschaft.

\section{References}

1. Yarden, Y. and Sliwkowski, M. X. (2001) Untangling the ErbB signalling network. Nat. Rev. Mol. Cell Biol. 2, 127-137.

2. Schlessinger, J. (2002) Ligand-induced, receptor-mediated dimerization and activation of EGF receptor. Cell 110, 669-672.

3. Gadella Jr., T. W. J. and Jovin, T. M. (1995) Oligomerization of epidermal growth factor receptors on A431 cells studied by time-resolved fluorescence imaging microscopy. A stereochemical model for tyrosine kinase receptor activation. J. Cell Biol. 129, 1543-1558.

4. Moriki, T., Maruyama, H., and Maruyama, I. N. (2001) Activation of preformed EGF receptor dimers by ligand-induced rotation of the transmembrane domain. J. Mol. Biol. 311, 1011-1026.

5. Sako, Y., Minoghchi, S., and Yanagida, T. (2000) Single-molecule imaging of EGFR signalling on the surface of living cells. Nat. Cell Biol. 2, 168-172.

6. Jorissen, R. N. Walker, F., Pouliot, N., Garrett, T. P., Ward, C. W., and Burgess, A. W. (2003) Epidermal growth factor receptor: mechanisms of activation and signalling. Exp. Cell Res. 284, 31-53.

7. Hynes, N. E., Horsch, K., Olayioye, M. A., and Badache, A. (2001) The ErbB receptor tyrosine family as signal integrators. Endocr. Relat. Cancer 8, 151-159.

8. Lidke, D. S., Nagy, P., Heintzmann, R., et al. (2004) Quantum dot ligands provide new insights into erbB/HER receptor-mediated signal transduction. Nat. Biotechnol. 22, 198-203.

9. Lidke, D. S., et al. (2005) Reaching out for signals: filopodia sense EGF and respond by directed retrograde transport of activated receptors. J. Cell Biol. 170, 619-626.

10. Grecco, H., Lidke, K. A., Heintzmann, R., et al. (2004) Ensemble and single particle photophysical properties (two-photon excitation, anisotropy, fret, lifetime, spectral conversion) of commercial quantum dots in solution and in live cells. Micros. Res. Tech. 65, 169-179.

11. Mattoon, D., Klein, P., Lemmon, M. A., Lax, I., and Schlessinger, J. (2004) The tethered configuration of the EGF receptor extracellular domain exerts only a limited control of receptor function. Proc. Nat. Acad. Sci. USA 101, 923-928.

12. So, M. K., et al. (2006) Self-illuminating quantum dot conjugates for in vivo imaging. Nat. Biotech. 24, 339-343.

13. Alivisatos, A. P., Gu, W. W., and Larabell, C. (2005) Quantum dots as cellular probes. Ann. Rev. Biomed. Eng. 7, 55-76.

14. Gao, X. H., et al. (2005) In vivo molecular and cellular imaging with quantum dots. Cur. Opin. Biotech., 16, 63-72.

15. Smith, A. M., et al. (2006) Engineering luminescent quantum dots for In vivo molecular and cellular imaging. Ann. Biomed. Eng. 34, 3-14. 